\title{
HOSPITAL-LEVEL VARIATION IN THE QUALITY OF BENIGN INPATIENT UROLOGIC SURGERY
}

\author{
Hilary Zetlen, MPH ${ }^{1}$, Kenn B. Daratha, PhD $^{2}$, Jonathan D. Harper, MD $^{1}$, Hunter Wessells, \\ MD ${ }^{1}$, Kenneth P. Roberts, $\mathrm{PhD}^{3}$, and John L. Gore, MD, MS ${ }^{1}$ \\ ${ }^{1}$ Department of Urology, University of Washington School of Medicine, Seattle, WA \\ ${ }^{2}$ College of Nursing, Washington State University Spokane, WA \\ ${ }^{3}$ College of Medical Sciences, Washington State University, Spokane, WA
}

\begin{abstract}
Objective-To examine hospital-level variation in outcomes following benign urologic surgeries given that hospital level variation in surgical outcomes can portend quality and appropriateness of care concerns and identify quality improvement opportunities in perioperative care.
\end{abstract}

\begin{abstract}
Methods-Using the Washington State Comprehensive Hospital Abstract Reporting System (CHARS), we identified patients that underwent transurethral resection of the prostate (TURP), percutaneous nephrostolithotomy (PCNL), and pyeloplasty from 2003-2008. We classified prolonged postoperative length of stay (LOS) as that exceeding the $75^{\text {th }}$ percentile, and we measured the rate of Agency for Healthcare Quality Patient Safety Indicators (PSIs), readmissions, and death. We calculated hospital-specific observed-to-expected event rates using random effects multilevel multivariable models adjusted for age and comorbidity.
\end{abstract}

Results-We identified 6,699 TURP patients at 54 different hospitals, 2,541 PCNL patients at 45 hospitals, and 584 pyeloplasty patients at 36 hospitals. Complication rates were highest after PCNL (22.9\% prolonged LOS vs. $17.3 \%$ for TURP and $13.9 \%$ for pyeloplasty, $\mathrm{p}<0.001 ; 3.4 \% 90$ day mortality vs. $0.6 \%$ for TURP and $0 \%$ for pyeloplasty). Hospital-level variation was most substantial for LOS after TURP and pyeloplasty ( $8.1 \%$ and $14.3 \%$ of variance in prolonged LOS, respectively).

Conclusions-Hospital-level variation is common after benign inpatient urologic surgeries and may relate to difference in perioperative provider practice patterns. The morbidity of PCNL in this study was higher than expected and merits further investigation.

\section{Keywords}

quality of care; variations in care; percutaneous nephrostolithotomy; transurethral resection of prostate; pyeloplasty

Corresponding Author: John L. Gore, MD, MS, Assistant Professor, Department of Urology, University of Washington School of Medicine, 1959 NE Pacific St, Box 356510, Seattle, WA 98195, Fax: 206-543-3272, Phone: 206-221-6430, jlgore@u.washington.edu.

Publisher's Disclaimer: This is a PDF file of an unedited manuscript that has been accepted for publication. As a service to our customers we are providing this early version of the manuscript. The manuscript will undergo copyediting, typesetting, and review of the resulting proof before it is published in its final citable form. Please note that during the production process errors may be discovered which could affect the content, and all legal disclaimers that apply to the journal pertain. 


\section{INTRODUCTION}

There is a growing body of research demonstrating significant variation in patient outcomes following urological surgeries ${ }^{1}$. This variation in morbidity and mortality may be due to regional differences in practice standards, health care delivery, or other, as yet to be determined factors that affect both provider and patient preferences and processes of care ${ }^{2}$. From a quality improvement perspective, the apparent differences in morbidity and mortality following common, benign surgical procedures indicate that even these straightforward and theoretically less complicated surgeries are susceptible to misuse, under or overuse, or other non-evidence-based practice variation that may have an observable affect on patient outcomes. There is a need to examine and understand this variation in outcomes in order to target quality improvement efforts to improve patient safety, and, secondarily, encourage ongoing transparency in reporting of surgical outcomes for greater understanding of practice variation.

There is a limited amount of literature examining benign urological procedure outcomes, although marked regional variation in utilization of transurethral resection of the prostate (TURP) in the treatment of benign prostatic hypertrophy has previously been observed ${ }^{3}$. In looking at other commonly performed urological procedures, there is evidence that selection of surgical approaches to pyeloplasty in adults with ureteropelvic junction obstruction is influenced by patient insurance status, physician volume, and hospital type; there are also significant sociodemographic disparities in pyeloplasty treatment selection that may influence surgical outcomes ${ }^{4,5}$. Likewise for percutaneous nephrostolithotomy (PCNL), patient disposition has been shown to vary with hospital volume, further supporting the hypothesis that certain hospital- or institutional-level factors may affect patient outcomes in unexpected ways ${ }^{6}$. There is evidently a need for closer analysis of surgical outcome variation and its antecedents in urological practice.

We utilize the Washington Comprehensive Hospital Abstract Reporting System (CHARS) to evaluate hospital-level variation in morbidity and mortality following TURP, PCNL, and pyeloplasty in an effort to better characterize the impact of practice variation on patient outcomes. The benefit of using a statewide comprehensive surgical abstract reporting system to examine patient outcomes following common, benign procedures is that the dataset encompasses a patient population with sufficient breadth and diversity to provide meaningful insight into hospital-level variation in outcomes across the State.

\section{MATIERIALS AND METHODS}

\section{Study sample}

We retrospectively analyzed a cohort of patients undergoing benign inpatient urological surgery in Washington State between 2003 and 2008. We selected surgeries based on the most prevalent inpatient surgeries for benign urological pathologies as determined by the Urologic Diseases in America Project ${ }^{7}$. CHARS contains data from patients admitted to Washington State hospitals for medical diagnoses and surgical procedures. Linked encrypted patient identification numbers allow abstraction of subsequent readmissions and 
reoperations, including those occurring at Washington hospitals other than the hospital where the initial surgery was performed. Site of care codes for each patient enable analysis of surgical outcomes by hospital.

We identified patients who underwent benign inpatient urological surgeries using relevant International Classification of Diseases, $9^{\text {th }}$ Edition (ICD-9) procedure and diagnosis codes. We identified the TURP cohort on the basis of procedure ICD-9 codes (ICD-9 code 60.29) in conjunction with an absence of a code for prostate cancer (ICD-9 code 185). Procedure ICD-9 code 55.04, in conjunction with the diagnosis codes for kidney or ureteral stones (ICD-9 codes 592.0, 592.1), was used to identify the PCNL cohort. The pyeloplasty cohort was identified on the basis of ICD-9 procedure code 55.87 for correction of UPJ obstruction. We restricted our sample to cases from hospitals that performed at least two surgeries for each procedure and limited our cohort to adults 18 years of age and older.

\section{Covariates}

We adjusted for the case mix differences between hospitals using demographic and clinical characteristics. Patient age was analyzed as a continuous variable as the patients who underwent the urological procedures have different age distributions. We enumerated the number of each patient's comorbid conditions using the Elixhauser method. ${ }^{8}$ We considered only the ICD-9 codes of the hospitalization in which the surgical procedure was performed in consideration of the patient comorbidity count. We also examined differences in primary payer for the surgeries analyzed. Primary payer was categorized into Medicare, Medicaid, private insurance (including health maintenance organizations), and other insurance (including self-pay and charity care). Patient race/ethnicity was not available in CHARS for the complete study period and thus was omitted from our analyses.

From individual hospital identifiers, we assessed hospital-specific procedure volume by hospital, and categorized hospitals into volume quartiles based on their 5-year collective TURP, PCNL, or pyeloplasty surgery volume. Volume has been previously shown to be positively associated with surgical outcomes for many urological procedures. ${ }^{9-12}$

\section{Outcome measures}

Our main outcomes were prolonged postoperative length of stay (LOS), the occurrence of postoperative adverse events measured using Agency for Healthcare Research and Quality (AHRQ) Patient Safety Indicators (PSIs), inpatient readmissions after discharge from the index surgery, and postoperative mortality. Prolonged LOS was defined by an admission duration that was greater than the $75^{\text {th }}$ percentile for the respective surgical procedure. Adverse events within 30 days of surgery as measured by AHRQ PSIs were abstracted using PSI software available from AHRQ. ${ }^{13}$ The PSI software identifies ICD-9 codes indicative of events such as postoperative hemorrhage and venous thromboembolic events. We examined 30-day readmissions for any admission assuming that all cause postoperative readmissions would be associated with the hospitalization in which the urologic procedure was performed. CHARS links to the Washington State death registry, facilitating ascertainment of 90-day all-cause mortality. We considered any death within 90 days of the procedure a postoperative mortality. 


\section{Statistical analysis}

Descriptive statistics were used to express the characteristics of our study sample; outcomes were analyzed as pooled cross-sectional observations. We used chi-squared analysis to assess differences in categorical variables by index surgery and analysis of variance to assess differences in continuous variables by index surgery. We assessed hospital-level variation in outcomes after TURP, PCNL, and pyeloplasty with random effects multilevel multivariable models. We selected this method based on prior work evaluating surgeon-specific mortality outcomes after cardiovascular surgery in New York and Massachusetts ${ }^{14,15}$ and previously used these methods to examine hospital-level variation in outcomes after urological cancer surgery. ${ }^{1}$ Individual-level patient variables represented the level one fixed effects in our models. Hospitals represented the level two random effects in our models. Covariates in the model included patient age and comorbidity count to adjust for case mix differences between hospitals. Post-estimation analysis using the calculated random intercepts for each hospital permitted calculation of predicted event rates for each hospital. Confidence intervals around estimates of the observed-to-expected ratio of postoperative adverse events were estimated through comparison of hospitals to the statewide average in outcomes. ${ }^{16} \mathrm{~A}$ hospital was considered an outlier in quality of care if their outcomes were better or worse than the statewide average at an alpha level of 0.05 . The residual intraclass correlation coefficient from the random effects multilevel multivariable models allowed calculation of the partitioned variance attributable to hospital-level factors for each outcome of each surgical procedure. ${ }^{17}$ Statistics were performed using SAS version 9.2 (SAS Institute, Cary, $\mathrm{NC})$.

\section{RESULTS}

Demographic and clinical characteristics differed by surgical cohort (Table 1). We identified 6,699 patients who underwent transurethral resection of the prostate (TURP) at 54 different hospitals, 584 patients who underwent pyeloplasty at 36 hospitals, and 2,541 patients who underwent percutaneous nephrostolithotomy (PCNL) at 45 hospitals. Accordingly, hospital volume for TURP procedures during the study period was significantly higher than both PCNL and pyeloplasty (Supplementary Figure). Patient age varied significantly among the three procedures $(p<0.001)$; the majority of patients in the TURP cohort underwent the procedure at age 70 or greater, whereas patients in the pyeloplasty cohort were, on average, much younger. Payer data for these patients corresponded to age distribution, with Medicare serving as the primary payer for the vast majority of patients in the TURP cohort, while Medicare was the primary payer for a minority of patients in the pyeloplasty cohort, most of who were covered by commercial insurance. Concomitant with procedural differences in patient age, prevalence of comorbid conditions also varied significantly among procedures, with the comparatively older patients in the TURP and PCNL cohorts bearing the highest burden.

Although adverse events, as defined by AHRQ PSIs, were rare across all procedures, thromboembolic events were significantly more common among PCNL patients $(p<0.001)$ than those undergoing TURP or pyeloplasty. Patients undergoing PCNL and, to a lesser degree, patients undergoing TURP were also significantly more likely to have a prolonged 
length of stay ( $>75^{\text {th }}$ percentile) than those undergoing pyeloplasty procedures $(p<0.001)$. Readmission rates were markedly higher following PCNL than following TURP or pyeloplasty $(p<0.001$ ), with nearly half of PCNL patients returning to the hospital within 90 days of the procedure; postoperative mortality within this same interval was also highest among patients undergoing PCNL.

Figure 1 demonstrates observed-to-expected ratios of length of stay outcomes following TURP, PCNL, and pyeloplasty. There is considerable variation in length of stay outcomes following TURP, including outlier hospitals that had lower and higher TURP volumes over the study period with higher than expected event rates. The hospitals with better than expected event rates were all high volume TURP centers. PCNL also showed variation in length of stay outcomes by hospital, though to a lesser degree than what was observed for TURP. Somewhat unexpectedly, higher volume hospitals demonstrated greater variation in length of stay outcomes following PCNL. Several high-volume hospitals had length of stay outcomes that were significantly better than the state average. There were no statistically significant differences in length of stay following pyeloplasty across hospitals. However, hospital-level factors significantly modified length-of-stay outcomes across hospitals for all procedures - the hospital-level proportions of variance for length of stay following TURP, PCNL, and pyeloplasty were $8.1 \%, 4.1 \%$, and $14.3 \%$, respectively.

Observed-to-expected event rates for various hospital-level morbidity and mortality outcomes following all three procedures are shown in Figure 2. 90-day readmission rates following TURP (Figure 2A) were largely uniform, although greater variation in readmission rates was observed among higher-volume hospitals. Mortality within 90-days of PCNL did not differ significantly across hospitals, although hospital-level mortality rates following PCNL showed considerably more variation than mortality outcomes following TURP or pyeloplasty. Variance in mortality outcomes following both TURP and PCNL appeared to be significantly associated with hospital-level differences, with hospital-level proportions of variance in mortality of $9.4 \%$ and $4.7 \%$, respectively. Pyeloplasty was not associated with significant differences in 90-readmission rates across hospitals (Figure 2C).

\section{COMMENTS}

TURP, PCNL, and pyeloplasty are benign inpatient urological procedures whose rates are increasing nationally ${ }^{4}, 18$. In Washington State, there is significant variability in procedural outcomes related to the hospital where the procedure was performed, a finding that suggests underlying practice variation affecting patients' quality of care. This is best illustrated by the substantial observed variation in length-of-stay outcomes across all procedures, and the significant proportion of variance in those outcomes due to hospital-level factors. Although the baseline characteristics of patients undergoing each of these procedures vary considerably, the presence of hospital-level variation across all procedures suggests that differences in providers consistently play a significant role in patient outcomes regardless of the procedure being performed. These important findings demonstrate marked heterogeneity in urological practice patterns throughout Washington State that affects morbidity, mortality, patient disposition and, as a result, health care cost Given the potential impact of these findings on the delivery of urological care, this topic merits further investigation. 
TURP, which was by far the most common of the benign urologic procedures performed in Washington State from 2003-2008, exhibited the most dramatic variation in length of stay outcomes across hospitals. Unexpectedly, higher-volume hospitals showed the most significant variability in length of stay following TURP, with the ten highest-volume hospitals performing either significantly better or worse than expected. In fact, the proportion of variance in these outcomes that was due to the hospitals themselves was greater than $8 \%$. Unmeasured clinical details, such as prostate size and whether this was a primary or secondary procedure, may have affected these results. Length of stay following PCNL and pyeloplasty also showed considerable variation in length of stay outcomes across hospitals, with a remarkable $14.3 \%$ of variation in length of stay following pyeloplasty explained by hospital-level factors. Comparing outcomes across procedures is difficult due to the widely disparate patient populations undergoing these procedures; however, in this context, the consistent presence of significant hospital-level length-of-stay variation among these disparate groups of patients is highly informative. This suggests that, beyond baseline differences in patients or the procedures themselves, there are inherent differences among the providers performing these procedures or the hospitals where they were performed that affect patient outcomes. This study finding is representative of provider variation that would benefit from a closer look, ideally with more granular data that would allow for more targeted hypothesis generation to uncover differences in procedures not evaluable with the available disposition, morbidity and mortality data available in CHARS.

This study also revealed a surprising level of morbidity and mortality for patients undergoing PCNL, with $40 \%$ of patients requiring readmission within 90 days as well as a surprising 90-day mortality rate of $3.4 \%$. That the hospital-level variation in morbidity and mortality was not significant may indicate that PCNL is associated with inherent morbidity irrespective of the operating surgeon experience. The variation in mortality among highervolume hospitals may be indicative of differences in practice patterns among hospitals that could be more strongly demonstrated with a greater volume of data. This variation also may illustrate differences in the baseline case mix and clinical severity characteristics of patients undergoing PCNL at individual hospitals that we were unable to adjust for in our model. Although risk adjustment was performed for age and number of comorbid conditions, several unmeasured factors-such as the characteristics of specific concomitant comorbidities, size and type of treated stone, history of infected stones, or history of prior ipsilateral percutaneous procedures-may drive these results. PCNL patients treated at higher-volume hospitals may have an inherent and unmeasured complexity. Further investigation of the unexpected morbidity and mortality of PCNL is needed.

There were many limitations to this study. We have discussed the need for more granular data on patient demographic and clinical characteristics that may be important determinants of quality and health care outcomes. This study has identified PCNL as an important target for further investigation, ideally through surgical quality collaboratives, which have been used in other diseases to identify quality improvement opportunities and conduct quality assurance. This more granular data would facilitate more valid risk adjustment and isolation of procedures where planned two stage procedures explain observed long lengths of stay or early readmissions. It is also important to note that our cohort was limited to patients undergoing procedures in Washington State, which restricted both the volume of analyzable 
procedures and the generalizability of our results. Also, CHARS is limited to inpatient data, which may underestimate the occurrence of adverse events that were managed on an outpatient basis and also limited our ability to correlate adverse events with underlying characteristics not coded in CHARS. Since Washington State contains many regional referral centers, our data also did not capture any adverse events that occurred after patients returned to their home states for follow up. Patients in Washington State may also have different disease characteristics than patients in other regions, limiting the generalizability of our findings. Finally, it should be acknowledged that the inherent differences in patient populations undergoing each of these three procedures limited our ability to compare outcomes across all procedures. This may have obscured more system-wide variation in urological practice patterns that was not procedure-dependent and could inform us about hospital- and state-level factors that affect surgical outcomes.

\section{CONCLUSIONS}

Despite these limitations, we identified notable variation in postoperative outcomes following inpatient benign urologic surgeries. This variation may portend important differences in the practice styles of urologists that may be associated with quality of care and increased health care costs. Rates of morbidity and mortality after PCNL in this populationbased cohort were surprisingly high and merit further study.

\section{Supplementary Material}

Refer to Web version on PubMed Central for supplementary material.

\section{Acknowledgments}

Research support: This work was supported by grant UL1RR025014 from the NIH National Center for Research Resources.

\section{References}

1. Gore JL, Wright JL, Daratha KB, et al. Hospital-level variation in the quality of urologic cancer surgery. Cancer. 2012; 118:987-96. [PubMed: 21792864]

2. Dodgion CM, Greenberg CC. Using population-based registries to study variations in health care. J Oncol Pract. 2009; 5:319-20. [PubMed: 21479073]

3. The Dartmouth atlas of health care, 1999. Chicago Ill: American Hospital Publishing; 1999.

4. Jacobs BL, Kaufman SR, Morgenstern H, et al. Trends in the treatment of adults with ureteropelvic junction obstruction. J Endourol. 2013; 27:355-60. [PubMed: 22967009]

5. Sukumar S, Sun M, Karakiewicz PI, et al. National trends and disparities in the use of minimally invasive adult pyeloplasty. J Urol. 2012; 188:913-8. [PubMed: 22819404]

6. Kadlec AO, Ellimoottil C, Guo R, et al. Contemporary volume-outcome relationships for percutaneous nephrolithotomy: results from the Nationwide Inpatient Sample. J Endourol. 2013; 27:1107-13. [PubMed: 23718230]

7. Litwin MS, Saigal CS, Beerbohm EM. The burden of urologic diseases in America. J Urol. 2005; 173:1065-6. [PubMed: 15758702]

8. Elixhauser A, Steiner C, Harris DR, et al. Comorbidity measures for use with administrative data. Med Care. 1998; 36:8-27. [PubMed: 9431328]

9. Begg CB, Cramer LD, Hoskins WJ, et al. Impact of hospital volume on operative mortality for major cancer surgery. JAMA. 1998; 280:1747-51. [PubMed: 9842949] 
10. Begg CB, Riedel ER, Bach PB, et al. Variations in morbidity after radical prostatectomy. N Engl J Med. 2002; 346:1138-44. [PubMed: 11948274]

11. Hollenbeck BK, Wei Y, Birkmeyer JD. Volume, process of care, and operative mortality for cystectomy for bladder cancer. Urology. 2007; 69:871-5. [PubMed: 17482924]

12. Hu JC, Gold KF, Pashos CL, et al. Role of surgeon volume in radical prostatectomy outcomes. J Clin Oncol. 2003; 21:401-5. [PubMed: 12560426]

13. Romano PS, Geppert JJ, Davies S, et al. A national profile of patient safety in U.S. hospitals. Health Aff (Millwood). 2003; 22:154-66.

14. Shahian DM, Torchiana DF, Shemin RJ, et al. Massachusetts cardiac surgery report card: implications of statistical methodology. Ann Thorac Surg. 2005; 80:2106-13. [PubMed: 16305853]

15. Daniels MJ, Gatsonis C. Hierarchical polytomous regression models with applications to health services research. Stat Med. 1997; 16:2311-25. [PubMed: 9351167]

16. Low RB, Ito S, Gregory R, et al. Using the GLIMMIX Procedure to Model Hospital Quality Measured by CMS: Comparing the City-Owned Hospitals, Other Safety Net Hospitals, and Hospitals for the Well Insured. SAS Global Forum. 2010

17. Snijders, TAB., Bosker, RJ. Multilevel analysis: an introduction to basic and advanced multilevel modeling. London; Thousand Oaks, Calif: Sage Publications; 1999.

18. Ghani KR, Sammon JD, Bhojani N, et al. Trends in percutaneous nephrolithotomy use and outcomes in the United States. J Urol. 2013; 190:558-64. [PubMed: 23434944] 


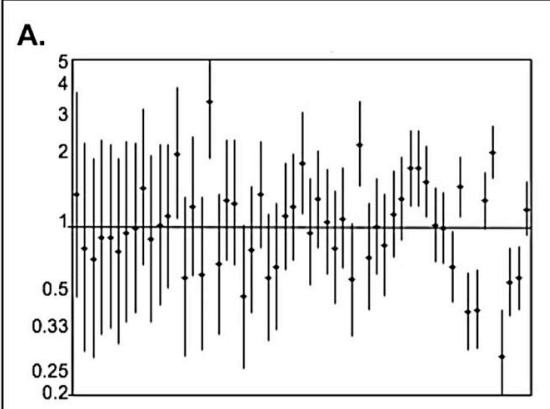

B.

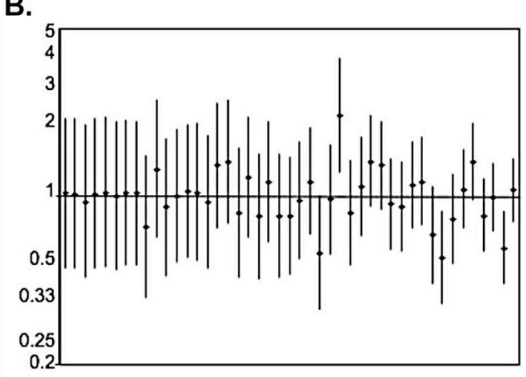

c.

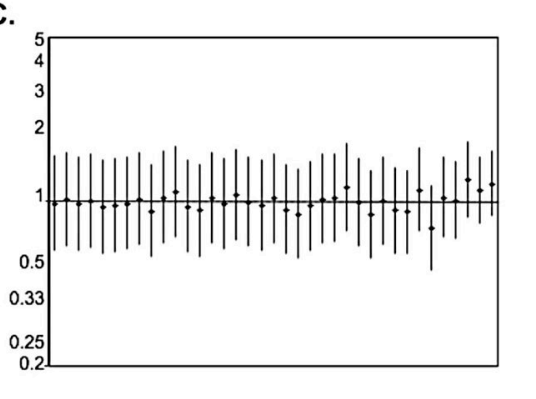

Figure 1.

Hospital volume is illustrated for the three study procedures, TURP (transurethral resection of the prostate), PCNL (percutaneous nephrostolithotomy), and pyeloplasty, ordered from hospital with lowest to highest TURP volume. 


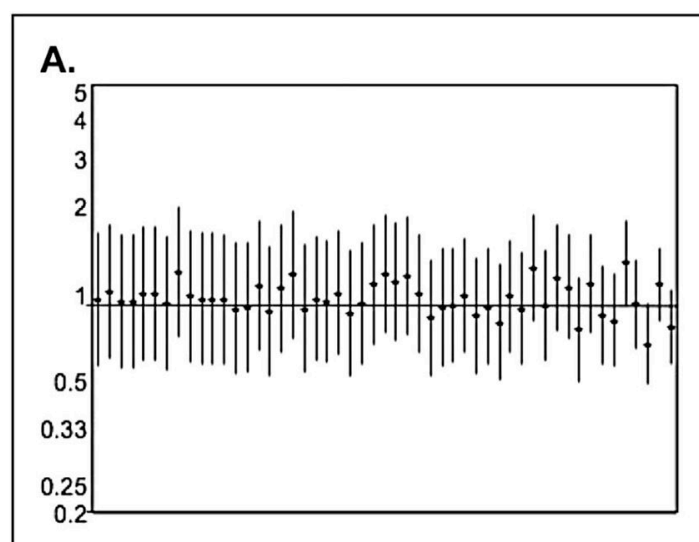

B.

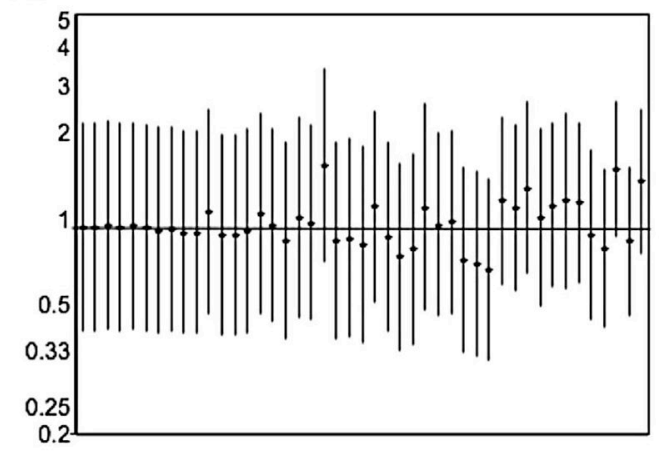

C.

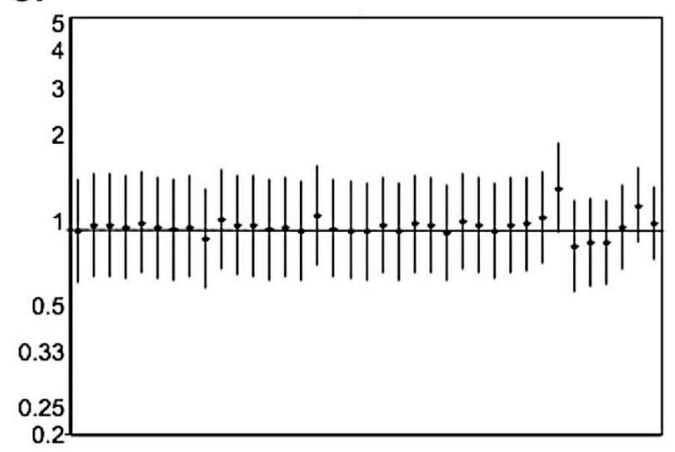

Figure 2.

Hospital-level variation is illustrated for prolonged length of stay ( $>2$ days) after (A) transurethral resection of prostate, (B) percutaneous nephrostolithotomy, and (C) pyeloplasty. Horizontal bars indicate the observed-to-expected ratio of events at each hospital, and vertical bars represent $95 \%$ confidence intervals. Hospitals are ordered according to procedure volume (from lowest to highest). 